\title{
Step-type stereo ecological seaborn construct and plant screening
}

\author{
Wang De-yong ${ }^{1,2,3, *}$, Wang Jing ${ }^{1,2,3}$, Zeng Qing-jun ${ }^{1,2}$, Deng Kai-fang ${ }^{1}$ \\ ${ }^{1}$ CCCC Fourth Harbor Engineering Institute Co.,Ltd, Guangzhou 510230,China; \\ ${ }^{2}$ Key Laboratory of Environmental Protection \& Safety of Communication Foundation Engineering, CCCC, Guangzhou 510230, China \\ ${ }^{3}$ Southern Marine Science and Engineering Guangdong Laboratory, Zhuhai 519082, China
}

\begin{abstract}
For the problem of traditional ecological seawall structure, ecological landscape monotonous problems, the stepped stereo ecological seawall integrating defense, ecological and landscape functions is proposed. In order to screen low-conditioned, salt, and suitable plants planted in coastal areas, 6 plants were studied in the form of sewage (Sonneratia, Arundo, Canna, Bischofia, Myriophyllum, Dianella) in different salt concentrations of salt sewage Change Law of Biochemical Indicators. The test results show that the chlorophyll content of Hazang, Mountains and Rusais increased significantly, and the content of malondialdehyde has a significant reduction in trend, indicating that they can thrive in salty sewage, have a high degree of stress resistance, and can be used as the preferred plants for ecological coast.
\end{abstract}

\section{Introduction}

Seafikes are an important infrastructure in the coastal defense typhoon storm disaster, ensuring economic and social development and the safety of the people's lives and property. At the same time, sea areas and coastlines are also scarce marine space resources ${ }^{[1]}$, and ecological construction of them is a necessary measure to implement the concept of ecological sea use, strengthen marine natural ecological protection, and build a strong ecological security barrier. As of 2018, my country's seabed coastline exceeded $15000 \mathrm{~km}$.

Long-term practice also found that traditional seawall construction has achieved significant mitigation and economic benefits, but it also has a certain impact on the environment ${ }^{[2]}$, such as: encroaching mangrove wetland, changing natural coastline and weakening biodiversity. Many developed countries put forward the construction ideas of ecological seabors, such as: The Netherlands put forward the ecological protection concept of "natural return nature", the United States complies with "Construction Following Natural" Protection Idea, Australia has "people and natural harmonious coexistence" Ideas, these philosophy and related measures significantly reduce human factors on the structure of ecological seabors, and provide the necessary space venues for the habitat of marine organisms.

On the basis of ensuring the physical protection of seawalls, enhancing the ecological value and landscape

*Corresponding author: de_yong_wang@163.com value of seawalls, and promoting coastal ecological construction, the construction of ecological seawalls is the main trend in the development of future marine engineering projects in my country ${ }^{[4]}$, and this model has shown its strength. Its vitality has become the first choice for future seawall project construction.

\section{Construction of stepped three-dimensional ecological seawall structure}

Ecological seawall construction is a long, continuous process, any factor in any factor, may have an effect of ecological seawall. The ecological seaborn be sure to assess the species diversity of the ecological seawall, fully investigate the degree of ecologicalization, and provide the necessary support for the construction of ecological seawall construction in the relevant regions.

The so-called ecological seawall should meet the three functions of physical engineering, ecological environmental protection and culturalities ${ }^{[5]}$ : First, physical resistance, disaster reduction and disaster prevention function is the main function of the seawall; secondly, the ecological seawall should try to keep or artificially creative In the natural vegetation and landscape elements, the original ocean life of the sea is as close as possible; in the end, the ecological seawall should meet the needs of local residents and relaxation. In order to ensure the beauty of the coastal ecology, the scientific criterion complied with the ecological seawall plan is: keep the coastal trend and terrain; try to retain and repair the original coast vegetation, construct the sea and land transition zone, the waters into the sea; greening 
try to use the local coastal plants, Eliminate biological invasion.

In combination in the current situation and demand of the Coast of South China, the new ecological seabark model integrating coastal protection, biodiversity retention, landscape optimization, and recreational, as shown in Figure 1-3.

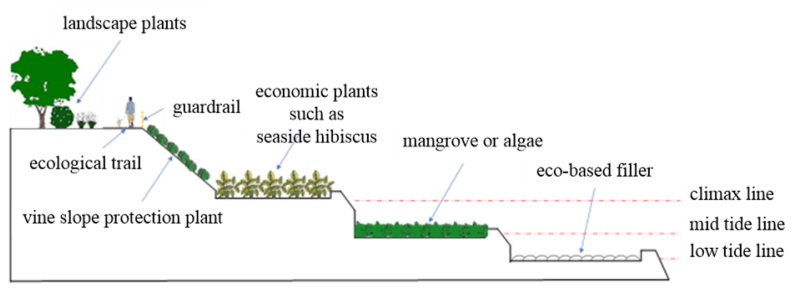

Fig.1 Stepped stereo ecological bed (mode 1)

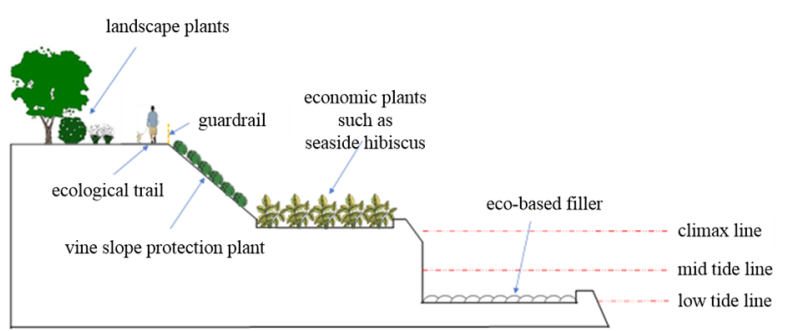

Fig.2 Stepped stereo ecological bed (mode 2)

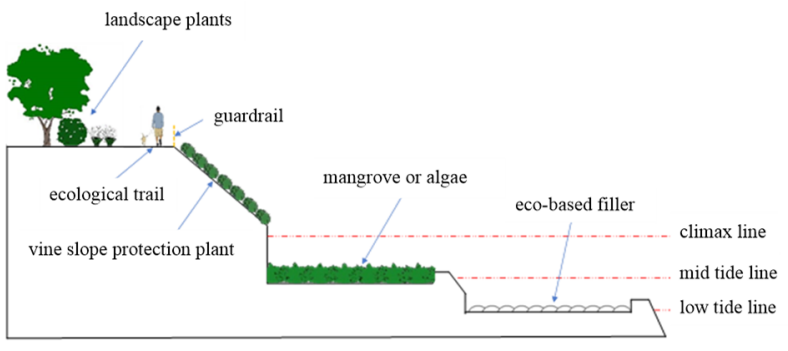

Fig.3 Stepped stereo ecological bed (mode 3)

\section{Optimum experiment of ecological seawall plants}

Screening of low-conditioned, salt, and plants that are suitable coastal areas, including Sonneratia, Arundo, Canna, Bischofia, Myriophyllum, Dianella 6 plants as tested plants. After three months of retrieving plant samples, all plant survival rates were $100 \%$, indicating that 6 plants have good water resistance. Thereafter, it is tested that the anti-counterpost is good, the growth is good, and the plant size is close to the plant.

Try the sewage for artificial preparation wastewater (formula is $3 \mathrm{~g}$ of milk powder, $0.5 \mathrm{~g}$ of dihydrogen phosphate, $0.5 \mathrm{~g}$ of sodium hydrogencarbonate, sodium bicarbonate $0.5 \mathrm{~g}$, sulfate $3.5 \mathrm{~g}$, soluble starch $3 \mathrm{~g}$, tap water $40 \mathrm{~L}$ ), The salted salt concentration is: $0,0.5 \%$, $1.0 \%, 1.5 \%, 2.0 \%$. Before the test start, the mid-test medium (3D) and the plant harvest (7d) were then determined after the plant physiological index chlorophyll, indoleacerate oxidase activity, and propylene derivatives.

Determination of plant chlorophyll content using acetone immersion method; Indoleacetic acid oxida-se; IAAO activity assay with colorimetric method; Malonaldehyde; MDA content determination of thiolansi Acid method.

\section{Test results and analysis}

The planting rate, growth condition is the shape of the adaptability of wetland plants, and the most direct reaction of the strength of plant adaptability. After a week of experiment, in the artificially prepared domestic sewage, the six plants selected were survived, indicating that the six plants had a certain water resistance. There is a significant difference in growth conditions, including Hanzang, Shannan and Lushu grow better.

Chlorophyll content and the photosynthetic ability of plants, the developmental growth phase is high, the correlation is obvious, and is one of the important indicators of monitoring the health of wetland vegetation. MDA is one of the main products of membrane fat oxidation, and is positively correlated with the relative perception of the cell membrane, the higher the content, the more serious the damage of the biofilm. The above six plants have been tested before and after tested in sternum water, as shown in Figures 4 to 7.

The test results show that the performance of 6 plants is different. Among them, sea Sang's chlorophyll content has increased, indicating that Heman is in good growth in salt stress; at the same time, the soluble sugar content has not increased, and the MDA content has decreased, and it is also shown that Hanzang is suitable for sewage in high salt concentration. Grow. It can be seen that Hai Sang is suitable as treated as a coastal slope plant or seawater breeding sewage. In addition, although the chlorophyll content of Shannan and Rushu is reduced, its growth is still good, this shows that mountain mailers and rut bamboo can maintain healthy growth in salt stress, or as a coastal slope plant or sea water Treatment of cultured sewage. Through the analysis of chlorophyll, MDA, soluble sugar content of 6 plants, combined with high, biomass, survival, etc., three plants such as Hazang, Mountain, Luzhu are more suitable for application in ecological coast construction. 


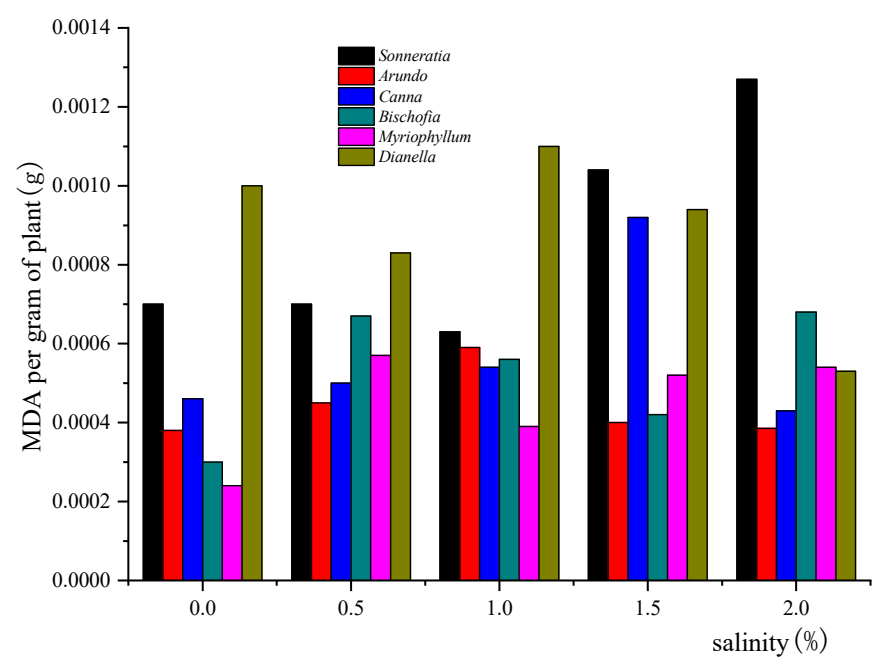

Fig.4 MDA per gram of each plant in new planting

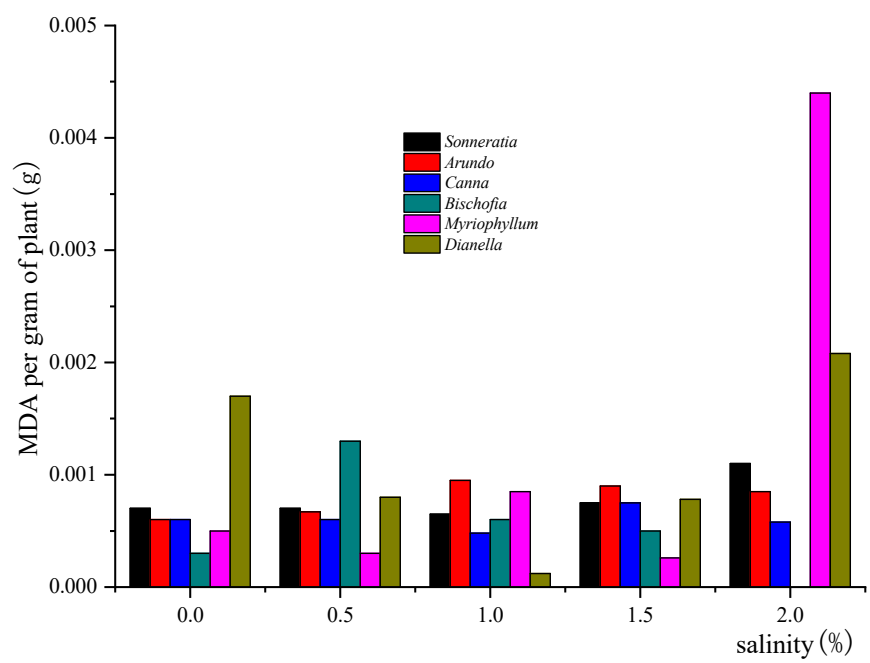

Fig.5 MDA per gram of each plant after growing in salt water

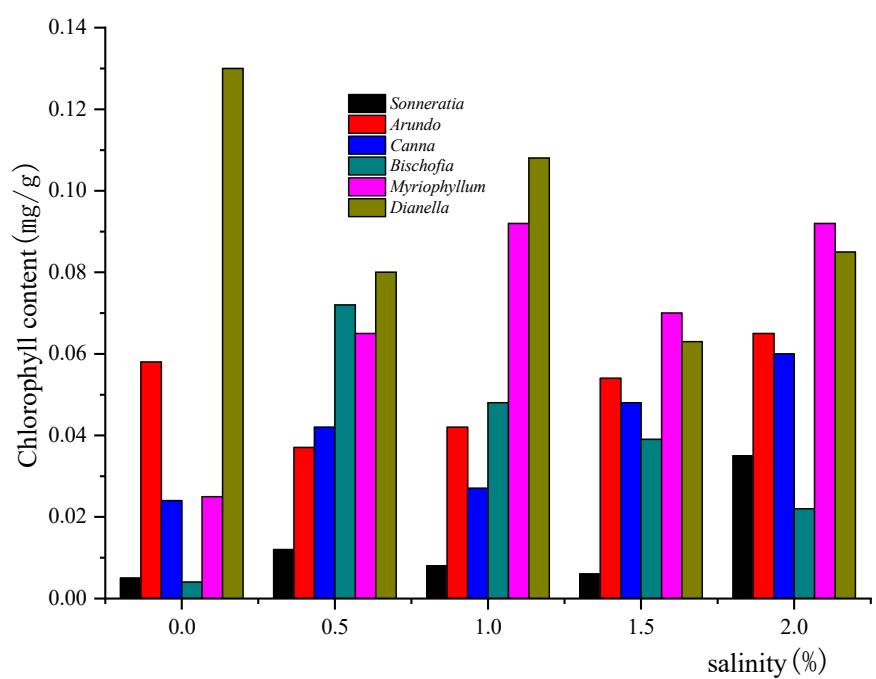

Fig.6 Chlorophyll content of each plant when newly planted 


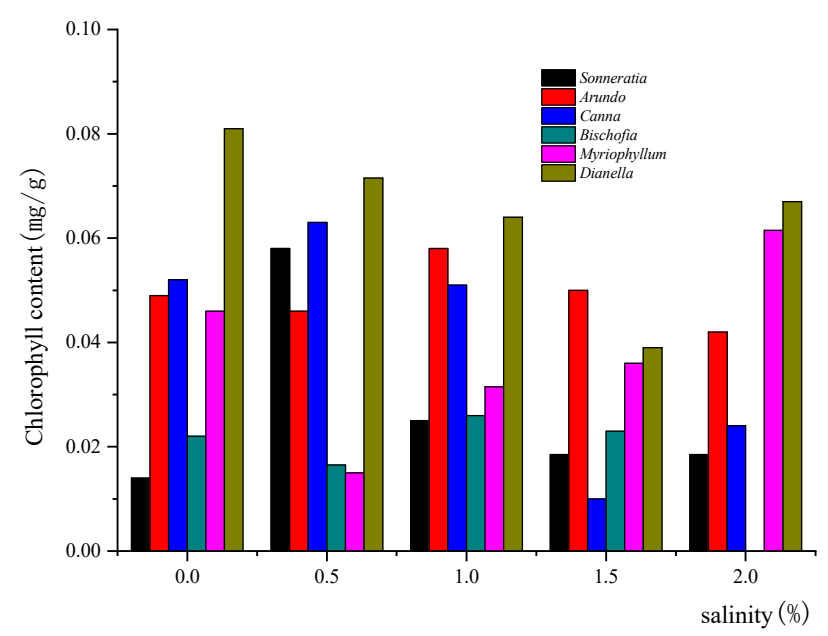

Fig.7 Chlorophyll content of each plant after growing in salt water

\section{Conclusion}

In view of the single function of the traditional ecological seawall structure and the monotonous ecological landscape, an ecological seawall structure that meets the three functions of physical engineering, ecological environmental protection and landscape culture was constructed, and combined with the characteristics of the southern ocean, the marine environment of six typical plants was studied. Adversity resistance, the main conclusions drawn are as follows:

(1) The new ecological seabark model integrating coastal protection, biodiversity retention, landscape optimization, and recreational leisure is constructed.

(2) 6 kinds of typical plants in the anti-reverse test of contaminated brine show that Haisang is in good growth in salt stress, and sea Sang is suitable as a coastal belt slope plant or seawater cultured sewage. Although the chlorophyll content of mountains and rhubarns decreased, the growth situation is still good, this shows that mountain mailer and rut bamboo can maintain healthy growth under salt stress, or as a coastal slope plant or seawater breeding sewage treatment.

\section{References:}

1. Zhu Zuhao, Jiang Hongyou, Li Li, etc. The development process and prospects of ecological seawalls, Ocean Bulletin, 2019, 38(5):481-490.

2. Liu Haiyang. Environmental Impact Analysis and Countermeasures of Guangdong Seawall Planning Project. Environmental Protection Science, 2003(06):50-52.

3. Xu Wei, Tao Aifeng, Liu Jianhui, et al. The enlightenment of international coastal ecological protection to the construction of ecological seawalls in my country. Ocean Development and Management, 2019, (10): 12-15.

4. Zhang Cuiping, Jia Houlei, Wu Lingling, et al. Research progress in seawall ecological construction technology and suggestions for promoting the ecological construction of seawalls in my country. Ocean Development and Management, 2020(9): 57-61.

5. Fan Hangqing, He Binyuan, Wang Xin, Concept and Practice of Ecological Seawall, Guangxi Science, 2017, 24(5): 427-434. 